\title{
Un sous-décalage du segment ST ? Non, une repolarisation atriale !
}

\section{ST Segment Depression? No, It's Atrial Repolarisation!}

\author{
H. Andrianjafy \\ (c) SFMU et Lavoisier SAS 2015
}

Une patiente de 46 ans consulte aux urgences pour épigastralgie et anxiété quelques jours après une chirurgie bariatrique par anneau gastrique. La patiente est tachycarde, et un ECG est réalisé (Fig. 1). Il s'agit typiquement d'une repolarisation atriale : l'onde $\mathrm{T}$ atriale débute à la fin de l'onde $\mathrm{P}$, a une polarité inversée par rapport à celle-ci et se poursuit audelà du QRS ; l'espace PR est court et descendant. La repolarisation atriale est rarement visible, mais sa transcription sur l'ECG doit être reconnue, car en cas de tachycardie comme lors d'un stress ou d'un exercice physique intense, on peut voir l'onde $\mathrm{T}$ atriale qui se prolonge après le QRS donnant ainsi un aspect sous-décalé du segment ST, ce qui pourrait faire suspecter à tort un trouble de repolarisation ventriculaire ischémique [1] et prescrire des examens complémentaires coûteux et un traitement inutile ou dangereux.

\section{Référence}

1. Sapin PM, Koch G, Blauwet MB, et al (1991) Identification of false positive exercise tests with use of electrocardiographic criteria: a possible role for atrial repolarization waves. J Am Coll Cardiol 18:127-35

H. Andrianjafy $(\square)$

Service des urgences, centre hospitalier Longjumeau, groupe hospitalier Nord-Essonne,

159, rue du Président-François-Mitterrand,

F-91160 Longjumeau, France

e-mail : h.andrianjafy@gh-nord-essonne.fr 